\title{
MANUTENÇÃO INDUSTRIAL EFICIENTE: UM ESTUDO DE CASO APLICANDO TERMOGRAFIA
}

\author{
Phellipe T. DinARDi ${ }^{1}$, KeVEN S. DA COSTA $^{2}$, ISRAEL G. TORNÉ ${ }^{1}$.
}

1. Laboratório de Sistemas Embarcados, HUB - Tecnologia e Inovação, Universidade do Estado do Amazonas

Caixa Postal 69050-020, Manaus, AM, Brasil

E-mails:hub_lse@uea.edu.br

\section{Depto. de Engenharia Elétrica, Universidade do Estado do Amazonas} Caixa Postal 69050-020, Manaus, AM, Brasil

E-mails: ptd.eng16@uea.edu.br, ksdcs.eng@uea.edu.br, igondrest@gmail.com

\begin{abstract}
The industry has constantly allied to the maintenance of its plant as a measure of optimization of profits, costs and development of advantages. The thermography proposes to be an improved tool in predictive maintenance. The aim of this paper is to study the applications of thermography in an industrial installation, verifying the time and efficiency of the tool. In an analysis of a system with a monthly average capacity of 165,652 kWh, thermal images were captured and correlated with the industrial maintenance philosophies and the maintenance sector reports of the plant. Performance was obtained with excellent agility in the method of monitoring and recording the main electrical system of the plant with the description of the defects, faults and conditioners discriminated using the thermography. For future work it is recommended to collect and cross-measure measurements of defective equipment, obtained by invasive methods and by thermographic method, to measure statistical reliability and operation data.
\end{abstract}

Keywords_ Application in Industry, Industrial Maintenance, Thermography.

Resumo- A indústria tem constantemente aliado a manutenção da sua planta como uma medida de otimização de lucros, custos e desenvolvimento de vantagens. A termografia propõe ser ferramenta aprimorada na manutenção preditiva, por isso o objetivo deste trabalho é estudar as aplicações da termografia em uma instalação industrial verificando o tempo e a eficiência da ferramenta. Em análise de um sistema, com consumo de energia mensal aproximado de 165.652 kWh, capturou-se imagens térmicas e correlacionou-se com as filosofias de manutenção industrial e os relatórios do setor de manutenção da planta. Obteve-se desempenho com excelente agilidade no método de monitoramento e registro do sistema elétrico principal da fábrica com descrição dos defeitos, falhas e condicionantes descriminados pelo uso da termografia. Para trabalhos futuros recomenda-se a realização de coleta e cruzamento de medições acerca de equipamentos defeituosos, obtidas por métodos invasivos e por método termográfico, para dimensionar confiabilidade estatística e dados de operação.

Palavras-chave— Aplicação na Industria, Manutenção industrial, Termografia.

\section{Introdução}

Desde 2007 a Comissão Europeia formou uma diretriz para regulamentar o desempenho energético dos edifícios na qual uma de suas medidas é os Certificados de Desempenho Energético, em inglês Energy Performance Certificates (EPCs), tornando-se obrigatório em muitos países da União Europeia (Arcipowska 2014). Em análise das ações legais e econômicas realizadas na União Europeia após 2007 estimasse que critérios e normas mais severos na investigação de eficiência energética e capacidade de poupar energia serão exigidos (FLIR 2015). No Brasil é previsto no Prontuário das Instalações Elétricas NR10, documento obrigatório para instalações elétricas principalmente em ambiente industrial e exigido por auditoria fiscal do Ministério do Trabalho e Emprego, o relatório de inspeção termográfica (Barros 2010).

Os termos termografia infravermelha e imagens térmicas, são termos comuns usados na visualização e quantificação de temperatura superficial, utilizando câmeras infravermelhas. Em indivíduos saudáveis, o padrão da temperatura superficial costuma se comportar de forma simétrica (Mercer 2014). A pesquisa do infravermelho limitou-se ao efeito de aquecimento provocado pela incidência dessa radiação em material, assim, seu prosseguimento dependeu em grande parte da evolução termométrica.

Recentemente, diversas pesquisas têm sido realizadas para enriquecer o universo termográfico e suas implementações tecnológicas como: Utilização de correntes parasitas para detecção de defeitos em matérias ferromagnéticos, método não destrutivo para teste que combina aquecimento por indução magnética e tecnologia térmica por infravermelho (Yan 2016); Previsão de corrente em uma subestação a fim de programar termografia para a qualidade do sistema, utilizando os dados de uma linha de subestação Sueca num período de 10 anos (Westerlund 2016); Monitoramento de equipamentos para falhas relacionadas à temperatura utilizando câmeras de termografia, onde observa que o governo dos Estados Unidos da América está implementando programas e leis para garantir que infraestruturas críticas tenham soluções de monitoramento e apresenta alternativa além do métodos tradicionais de monitoramento que exigem medições de pontos pré-selecionados (Wang 2015). 
Diversos setores industrias estão mostrando um interesse crescente na integração tecnológica da Industria 4.0 em suas plantas para desenvolver estratégias de vantagem competitiva e de manutenção preditiva. No entanto, muitas abordagens de pesquisa despontam que o desenvolvimento de sistemas de manutenção preditiva precisa de melhorias significativas em termos de redução de custos para monitorar equipamentos e monitorá-los com maior precisão (Sezer 2018).

\section{Filosofias de manutenção industrial.}

Segundo as normas brasileiras de regulamentação, manutenção é a combinação de ações técnicas e administrativas, incluindo supervisão, destinadas a manter ou recolocar um item em um estado no qual possa desempenhar uma função requerida (ABNT 5462 1994). É um ponto crucial para assegurar a eficiência da produção na indústria, pois a ocorrência de perturbações inesperadas leva à degradação do desempenho do sistema, ocasionando perda de produtividade e oportunidades de negócios. Porém, normalmente os custos associados às tarefas de manutenção em uma empresa de produção são extremamente significativos (Cachada 2018).

Relacionando os resultados obtidos aos custos de um sistema podemos estabelecer a eficiência do mesmo. E em análise a outros sistemas teremos, em média, um sistema mais eficiente que o outro, portanto esse processo produz mais, consumindo menos e com melhor qualidade (Santos 2007). O melhor índice de resultados na manutenção é o Valor Global da Eficácia de Equipamento, em inglês Overall Effectiveness of Equipment - OEE, que pondera a disponibilidade do equipamento, a diminuição no retrabalho, a rejeição e a taxa de desempenho.

Alguns teóricos apresentam a manutenção detectiva como a atuação efetuada em sistemas nos quais as falhas ou alterações no funcionamento regular do equipamento não pode ser percebido pela equipe de operação e de manutenção (Mainkar 2017), porém as manutenções frequentes em qualquer sistema industrial são a preventiva, corretiva e preditiva.

Após o surgimento de uma falha num sistema a execução esperada é a correção do defeito, a conservação deste ciclo de quebra e reparação é denominada manutenção corretiva. Sua execução está sujeita a aleatoriedade do aparecimento do defeito e contribui para a diminuição da vida útil do sistema (Camara 2012). Um método passivo e constante em um sistema, no qual objetiva reduzir a probabilidade de falha do mesmo, maximizando a longevidade da vida útil do sistema e o benefício de sua operação para a planta é designado de manutenção preventiva (Allen 2015).

A Manutenção preditiva, encontrada em alguns teóricos sobre o nome de manutenção baseada na condição, busca um condicionante para deflagra a manutenção do equipamento, ou sistema, evitando um processo de falha futuro. Utilizando métodos de monito- ramento como inspeção periódica para avaliar o correto funcionamento do equipamento/sistema ao longo da vida útil do mesmo. A limitação do método de manutenção está relacionada a disponibilidade técnica efetiva de monitoramento e o custo de sua implementação e continuidade (Nassar 2015).

Estratégias para corroborarem com o funcionamento de plantas industriais foram desenvolvidas ao redor do mundo com o passar do tempo e a integração do usuário da máquina no papel de manutenção foi sistematizada e apresentada ao universo industrial por meio do Instituto Japonês de Manutenção de Plantas sobre o nome de Manutenção Produtiva Total, em inglês Total Productive Maintenance - TPM. Seu objetivo propõe um projeto funcional de time para redução de perda de equipamento alvo mudando a compreensão das pessoas e seus hábitos de trabalho ao usar equipamentos, como a implementação de ferramentas instrucionais, enquanto se aproximam de uma performance de qualidade, restaurando e controlando a deterioração e corrigindo anormalidades (Vardhan 2015). Um estudo realizado em um fábrica de manufatura buscou quantificar a eficácia da manutenção com foco no processo do TPM, onde ao final o custo e a qualidade foram significativamente aprimorados, reduzindo e minimizando a deterioração e as falhas do equipamento resultando em um OEE entre 85\% a 90\% (Wakjira 2012).

\section{Termografia na prática industrial}

Muitas industrias tem percebido a importante conexão entre manutenção e confiabilidade, em consequência o foco das manutenções tem sido estudar, manter e melhorar a confiabilidade e regularidade de operação do sistema produtivo por meio de uma constantemente na busca por otimização na manutenção (Fogliatto 2011). Assim, é preciso que a manutenção deixe de ser eficiente para se tornar eficaz, ou seja, não basta apenas repara o equipamento ou instalação tão rápido quanto possível, mas é preciso manter a função do equipamento disponível para a operação, reduzindo a probabilidade de uma parada de produção não planejada (Kardec 2009).

A termografia integra um método preditivo, no qual é possível detectar o surgimento de defeitos desde suas etapas iniciais agregando qualidade ao processo de manutenção e tornando o procedimento uma oportunidade de diferenciação da concorrência e aumento da competitividade ao reduzir a quantidade de manutenções preventivas que não agregam valor no produto final e significam maiores custos reduzindo a margem de lucro (Lemos 2011).

Para captura térmica de uma imagem, gerada por uma câmera infravermelha, é importante observar as matérias que compões o cenário a ser fotografado bem como os objetos e o meio que está inserido pois cada uma dessas partes influenciarão as análises realizadas. A emissividade é uma propriedade radiativa de um objeto em relação a um corpo perfeitamente negro, no qual é o emissor perfeito. Se a energia emitida de um 
corpo negro é denotada como $\mathrm{W}_{\mathrm{bb}}$, e a emitida por um corpo normal na mesma temperatura for denotada como $\mathrm{W}_{\text {obj}}$, a relação entre esses dois valores é descrita como a emissividade (Bramson 1968).

$$
\varepsilon=\frac{W o b j}{W b b}
$$

Assim, a emissividade é um número entre 0 e 1 . Quanto melhor as propriedades radiativas do objeto, maior sua emissividade. Um objeto que tenha a mesma emissividade para todos os comprimentos de onda é chamado de corpo cinza, em inglês denominado grey body, apesar de a maioria dos corpos não serem nem corpos negros a emissividade varia com comprimento de onda.

Como a termografia opera somente dentro de faixas espectrais limitadas, muitas vezes é possível tratar objetos como corpos de cinza. Em qualquer caso, um objeto pode ter emitância que varia fortemente com o comprimento de onda é chamado de radiador seletivo. O vidro é um exemplo de um radiador muito seletivo, comportando-se quase como um corpo negro para determinados comprimentos de onda, ao passo que é oposto para outros comprimentos de onda (Dos Santos 2006).

Entre a câmera e o objeto está a atmosfera, que tende a atenuar a radiação devido a absorção por gases e espalhamento das partículas. A quantidade de atenuação depende fortemente do comprimento de onda da radiação. Apesar de a atmosfera normalmente transmitir luz visível muito bem, neblina, nuvens, chuva e neve podem nos impedir de ver objetos distantes. O mesmo princípio se aplica a radiação infravermelha onde a atenuação atmosférica impede a radiação total de um objeto atingir a câmera. Se não for aplicada correção para atenuação, a medida aparente a temperatura será gradativamente menor com a distância aumentada. O Software da câmera IR corrige a atenuação atmosférica.

Uma análise bem sucedida no ensaio termográfico deve ser feito em condições atmosféricas/ambientias mais proximas do ideal possivel, devem ser tomados cuidados em relação aos equipamentos que serão estudados, por exemplo transformadores, compressores, grupos geradores, nesse tipo de equipamento deve ser feita a termografia durante os ciclos de trabalho dos mesmos, vale salientar que alem dos fatores ambientais e climáticos devemos levar em consideração o aspecto humano, o profissional que irá realizar o ensaio deve ser treinado, ou minimamente instruido, para que todas as precauções citadas anteriormente sejam consideradas durante a realização do ensaio (Snell 1992).

As radiações que atingem as lentes da câmera originam-se de três fontes diferentes e possuem suas fórmulas de absorção expressas pelo fabricante. A câmera recebe radiação do objeto de estudo, do se entorno e do reflexo da superfície. Utilizando a equação 2 podemos obter uma formula que calcula a temperatura de um objeto que teve sua imagem capturada por uma câmera termográfica (FLIR 2015).

$$
E O=\varepsilon \times \mathrm{T} \times W o b j
$$

Onde "EO” é a emissão do objeto, $\varepsilon$ é a emissividade do objeto e T é a transmitância da atmosfera.

De forma similar a energia de uma fonte presente no ambiente será fotografada pela câmera e deve ter seu valor considerado para não interferir na correta medição de temperatura do objeto. Seu comportamento é descrito na fórmula seguinte.

$$
E F=(1-\varepsilon) \times \mathrm{T} \times W a m b
$$

Onde "EF" é a emissão refletida da fonte e (1-E) é a refletância do objeto. E assumimos que a temperatura "W $\mathrm{amb}_{\text {a }}$, é a mesma para toda superfície emissora.

Por fim, o meio entre a câmera e o objeto interage com a radiação liberada e emissão da atmosfera atenua ou agrava a radiação capturada. Pode ser calculada com a equação 4.

$$
E A=(1-\mathrm{T}) \times \text { Watm }
$$

Onde "EA" é a emissão da atmosfera, (1-T) é a emissividade da atmosfera.

O somatório de todas as emissões que chegam a lente da câmera resulta na potência de radiação total apresentada na imagem capturada e pode ser escrita na equação abaixo.

$$
\text { Wtotal }=E O+E F+E A
$$

Ajustando à temperatura correta, o software da câmera requer como entrada a emissividade do objeto, atenuação atmosférica e de temperatura, e a temperatura do entorno. Dependendo das circunstâncias, esses fatores devem ser medidos, assumidos ou encontrados em uma tabela apresentada pelo fabricante, mas este processo de calibragem da câmera assegura qualidade nas interpretações possíveis com as imagens termográficas.

\section{Materiais e Métodos}

Realizou-se visita técnica nas instalações elétricas de distribuição, fotografando quadros de distribuição geral, possuindo sigla de QGD, e quadros gerais de baixa tensão, também denominado de QGBT, bem como a subestação composta por dois transformadores de $500 \mathrm{kVA}$, um transformador de $300 \mathrm{kVA}$ e um transformador de 112,5 kVA além dos cabos de muflas. A média mensal do consumo de energia elétrica do sistema visitado no ano de 2018 é de 165.658 kWh.

O sistema possui alimentação radial simples, no qual o fluxo de potência tem sentido único da fonte para a carga. É o tipo mais simples de alimentação industrial e também o mais utilizado (Mamed Filho 2013). 


\subsection{Etapa Estratégica}

Em contato com a equipe de manutenção das instalações elétricas obteve-se a rotina de manutenção do sistema e as falhas ocorridas nos últimos três meses bem como as investigações e métodos de mitigação das falhas existentes.

A equipe transmitiu completa ausência de práticas preditivas sendo $87 \%$ das atuações realizadas nos últimos três meses com carácter corretivo e o restante em medidas preventivas. A equipe, composta de seis funcionários, relataram aleatórios acionamentos do sistema de proteção em um dos blocos do sistema.

\subsection{Inspeção termográfica}

Após levantamento de dados de consumo e dos equipamentos ligados ao sistema objetivou-se a captura de imagens térmicas dos principais sistemas de distribuição de energia elétrica onde nossa atuação se deu da entrada da subestação até os quadros de distribuição geral. Obtidas as imagens efetuou-se o tratamento das imagens e a calibração dos parâmetros apresentados na equação 5 para assegurar confiabilidade nas informações obtidas da instalação.

\subsection{Análise de Dados}

Por fim, aplicou-se uma analise quantitativa e qualitativa das imagens obtidas para filtra-las selecionando os problemas recorrentes encontrados. Correlacionou-se as falhas relatados pela equipe de manutenção com os condicionantes encontrados pela termografia avaliando o funcionamento do sistema e suas possíveis futuras falhas.

\section{Resultados e Discussões}

Após visita pelas instalações e compartilhamento de informações com o setor de manutenção procedeuse com inspeção nas três subestações existentes, sendo uma delas desabrigada, nos cinco quadros gerais de baixa tensão e nos diversos quadros de distribuição geral. Num período de quatro horas foi possível monitorar todos o sistema existente com exceção dos maquinários e duas horas foram necessárias para tratar as imagens.

Concluida a etapa de aquisição dos termogramas passamos para a análise na qual os fatores técnicos citados anteriormente devem ser levados em consideração, e a partir de valores absolutos podemos partir para duas linhas de análise; um estudo quantitativo que baseia-se em valores de temperatura de trabalho tabelados, e uma análise qualitativa que no geral é feita de forma comparativa, comparando objetos trabalhando teoricamente nas mesmas condições, mas que apresentam discrepancias em seus valores absolutos de temperatura

Os transformadores apresentaram-se em temperatura normais de funcionamento e os últimos relatórios de resistência ôhmica, isolação, relação de transformação e análise do óleo também se apresentaram dentro da normalidade garantindo ao sistema vida útil mínima de 10 anos.

Na subestação abrigada observou-se condicionante de investigação, possivelmente falha na qualidade da energia entregue pela concessionária, no qual necessita de minucioso estudo das causas tendo em vista poderem prejudicar o bom funcionamento de qualquer planta industrial conforme apresentado na figura abaixo.

A figura 1 mostra os cabos de entrada de um transformador em uma cabine primária onde, após análise qualitativa, nota-se que no ponto em destaque, o ponto antes do isoladores tipo pedestal, temos um aquecimento que teoricamente é aceitável para o tipo de material, afinal, trata-se de um condutor rígido, dimensionado para uma tensão de operação de $13,8 \mathrm{kV}$, no entanto, como pode ser visto na imagem se comparamos as temperaturas de duas fases, vemos uma diferença de $43.9^{\circ} \mathrm{C}$ entre uma fase e outra, o que pode representar um grave erro de desbalanceamento de carga, bem como alguma falha nas operações do transformador ou presença de harmônicos, algo que representa um perigo eminente para a segurança dos indivíduos que estiverem próximos a esse local e dos equipamentos ligados a essa rede.

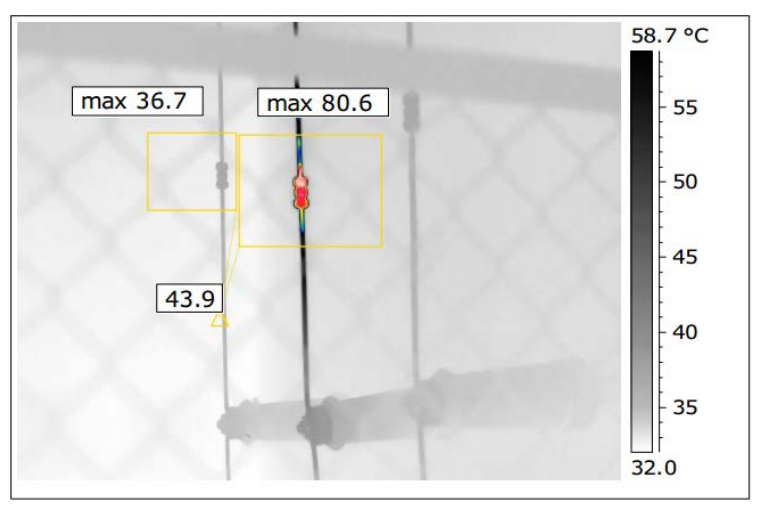

Figura 1. Termografia qualitativa

Os quadros gerais de baixa tensão apresentaramse regulares no funcionamento e segundo a equipe de manutenção não proporcionaram problemas nos últimos 5 anos. Com as imagens térmicas foi possível observar que apesar do funcionamento regular expansões de projetos ou ampliações de maquinários deverão ocasionar em falhas. A figura 2 apresenta uma temperatura diferenciada nos fusíveis de 400 A de um QGBT, tal como descrito na equação 1, sendo necessário retrofit. O quadro necessitou ser documentado e estabeleceu-se um plano de acompanhamento e avaliação do seu funcionamento e das cargas instaladas nele para evitar falhas futuras no fornecimento destes circuitos. 


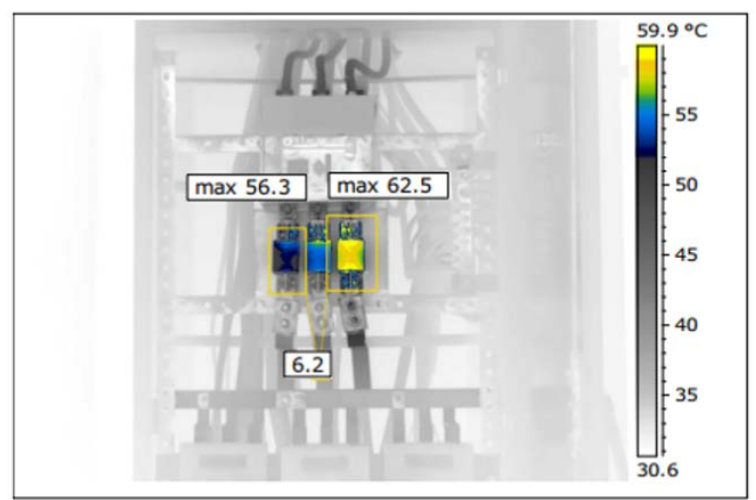

Figura 2. Termografia em QGBT

Por meio de inspeção visual foi possível constatar que os quadros gerais de distribuição possuíam diversas expansões, não prevista no projeto inicial, das quais alteravam o correto funcionamento do quadro colocando em risco a segurança dos usuários e aumentando a probabilidade de acionamento do sistema de segurança da linha. A maior parte dos quadros de distribuição geral apresentou algum aquecimento em tomadas de uso específica, mas dentro da faixa de temperatura de trabalho, e desbalanceamentos de carga com sobrecarregar constante na fase ' $\mathrm{S}$ '.

Durante o primeiro contato com o setor de manutenção do sistema foi apresentado aos pesquisadores o constante acionamento do sistema de proteção de um bloco secundário paralisando a linha de produção dependente daquele fornecimento, ocorrendo sempre nos momentos de pico de utilização e estando a equipe a duas semanas aferindo a causa da falha. Após captura termográfica do quadro de distribuição geral que fornece energia para a construção observou-se aquecimento irregular no cabeamento de alimentação do disjuntor geral conforme demonstrado abaixo.

Na figura 3 temos um exemplo de uma conexão a parafuso de um disjuntor, e um cabo ligado a ela com revestimento de PVC, nesse termograma podemos fazer uma análise levando em consideração valores de mercado para esse tipo de cabo, e conexão a parafuso, olhando para a imagem podemos ver que a temperatura de pico de $74,6^{\circ} \mathrm{C}$ se encontra de fato no parafuso, pois é o ponto de maior destaque, nesse ponto a temperatura máxima é de $120^{\circ} \mathrm{C}$, no entanto no cabo com revestimento $\mathrm{PVC}$ a temperatura máxima de trabalho é de $70^{\circ} \mathrm{C}$, sendo esses $74,6^{\circ} \mathrm{C}$ a temperatura de entorno do cabo vemos um risco potencial, que deve ser estudado com bastante atenção para evitar possíveis danos aos equipamentos interligados a esse circuito e falhas em momentos críticos da operação do sistema.

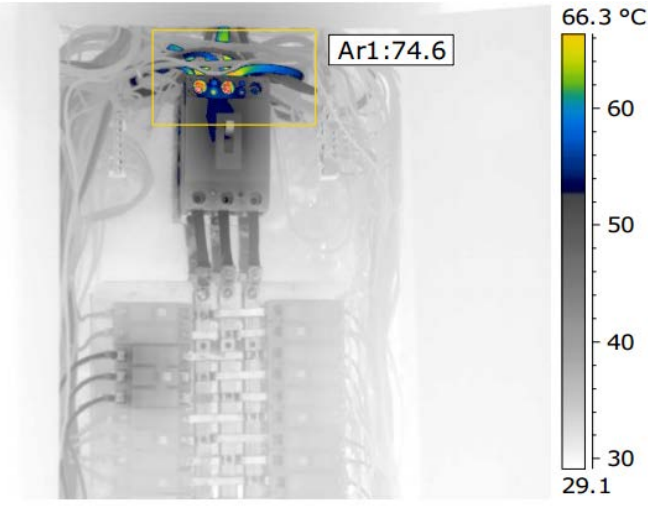

Figura 3. Termografia quantitativa

Através da termografia identificou-se sintomas térmicos que possibilitaram identificar o real problema do mau funcionamento de um dos sistemas de proteção; o desbalanceamento de carga generalizado em mais de $80 \%$ dos quadros de distribuição geral pode ser aferido de forma rápida e precisa com destaque para as fases desbalanceadas em cada quadro; temperatura elevada em uma das fases do barramento primário da subestação apresentou diferença de $43,9^{\circ} \mathrm{C}$ necessitando estudo mais aprofundado em harmônicos da rede e na qualidade da energia fornecida pela concessionária além de se recomendar o estudo de massa pela concessionária.

\section{Conclusões}

Em meio a necessidade de tornar a manutenção mais eficiente e eficaz o estudo demonstrou que a termografia é um excelente método de monitoramento preditivo ao identificar, de forma não invasiva sem necessário desligamento do sistema, condicionantes que deflagrem uma atividade de prevenção ou correção de acordo com o identificado. Realizou-se uma avaliação geral no sistema elétrico com rápida execução e pequena equipe de pessoal.

Em alguns casos do estudo foi possível detectar sistemas que não possuíam problema aparente, mas dos quais o potencial de falha ao sistema era grande. No total verificou-se 11 irregularidades responsáveis por falhas na qual 5 foram de baixo risco sendo sugerido acompanhamento, 4 de médio risco com necessárias soluções preventivas e 2 de risco imediato apresentando urgência de correção. Observa-se que a integração de atividade termográfica na equipe de manutenção é otimizadora na eficiência de uma indústria gerando alta produtividade, excelência em qualidade, baixos custos, e precisão no tempo de manutenção despendido.

Para trabalhos futuros recomenda-se a realização de coleta e cruzamento de medições acerca de equipamentos defeituosos, obtidas por métodos invasivos e por método termográfico, para dimensionar confiabilidade estatística e dados de operação. Um estudo aprofundado sobre a sensibilidade termográfica a baixos índices de qualidade de energia e seus impactos nos equipamentos também devem ser executados. 


\section{Referências Bibliográficas}

ALLEN, W. "Preventative maintenance and root cause analysis for critical process conveying equipment," 2015 IEEE-IAS/PCA Cement Industry Conference (IAS/PCA CIC), Toronto, ON, 2015, pp. 1-13

ARCIPOWSKA, A et all. Energy performance certificates across the eu - BPIE 2014.

ASSOCIAÇÃO BRASILEIRA DE NORMAS TÉCNICAS. NBR 5462 - Confiabilidade e mantenabilidade - Rio de Janeiro 1994.

BARROS, B. F. et all. NR-10 Norma Regulamentadora de Segurança em Instalações e Serviços de Eletricidade: Guia Prático de Análise e Aplicação. $1^{\text {a }}$ Edição. São Paulo: Érica, 2010.

CACHADA, A. et al. Maintenance 4.0: Intelligent and Predictive Maintenance System Architecture IEEE 23rd International Conference on Emerging Technologies and Factory Automation (ETFA) 2018.

CAMARA, J. et all. Manutenção elétrica industrial UFRN 2012.

DOS SANTOS, L. Termografia Infravermelha em Subestações de Alta Tensão Desabrigadas - Universidade Federal de Itajubá 2006.

FLIR. The Ultimate infrared handbook for R\&D Professionals - FLIR 2015.

FOGLIATTO, F. et all. Confiabilidade e manutenção industrial - Rio de Janeiro, ABEPRO 2011.

KARDEC, A. et al. Manutenção, função estratégica Rio de Janeiro, QUALITYMARK 2009.

LEMOS, M. et all. Qualidade na manutenção - ENEGEP 2011.

MAINKAR, M. S. and RATHOD, R. K. "Maintenance Management System Effective Tool to Progress and Reach Total Productive Maintenance \& Six Sigma Business Development Strategies". 2017 International Conference on Industrial Engineering, Management Science and Application (ICIMSA), Seoul, 2017, pp. 1-4.

MAMED FILHO, J. Instalações Elétricas Industriais - Rio de Janeiro, LTC 2013.

MERCER, J. et al. Thermography and thermal symmetry - IEEE International Symposium on Medical Measurements and Applications (MeMeA) 2014.

NASSAR, W. manutenção de máquinas e equipamentos - Universidade de Santa Cecília 2015.

SANTOS, A. et. al. Eficiência Energética Teoria \& Prática - PROCEL Educação, Universidade Federal de Itajubá 2007.

SEZER, E. et al. An Industry 4.0-enabled Low Cost Predictive Maintenance Approach for SMEs: A Use Case Applied to a CNC Turning Centre - IEEE International Conference on Emerging Technologies and Factory Automation (ETFA) 2018.

SNELL, J. et al. Developing Operational Protocol for Thermographic Inspection Programs, SPIE Vol. 1682, 1992.
VARDHAN, S. et al. "The impact of Quality Maintenance Pillar of TPM on manufacturing performance", 2015 International Conference on Industrial Engineering and Operations Management (IEOM), Dubai, 2015, pp. 1-6.

WANG, Y. et al. Monitoramento de equipamentos para falhas relacionadas à temperatura utilizando câmeras de termografia - IEEE Petroleum and Chemical Industry Committee Conference (PCIC) 2015.

WAKJIRA, W. et al. Melesse. Total Productive Maintenance: A Case Study in Manufacturing Industry - Global Journal of Research In Engineering 2012.

WESTERLUND, P. et al. Prediction of current in a substation in order to schedule thermography - IEEE International Conference on Probabilistic Methods Applied to Power Systems (PMAPS) 2016.

YAN, H. et al. Research on the detection mechanism of defect in ferromagnetic materials using eddy current thermography - IEEE International Conference on Ubiquitous Robots and Ambient Intelligence (URAl) 2016. 